\title{
Heart rate, defecation and genetic differences in rats
}

Maudsley reactive and nonreactive rats were tested for defecation and heart rate when handled at a home cage and in open field. The complex interrelationships found suggest several theoretical alternatives.

Following an earlier observation by Yoshioka (1932), Hall (1934) first used defecation in an open field as a measure of emotionality in the rat. It has since become the standard and accordingly has been both criticized (Bindra \& Thompson, 1958; Hunt \& Otis, 1953; O'Kelly, 1940; Lebo, 1953) and defended (Lindzey, Winston, \& Whitney, 1964; Pare, 1964)。

Selective breeding on defecation has revealed a substantial genetic component of this behavior (Hall, 1951; Broadhurst, 1960). A number of studies have followed on variables correlated with the differentiation of strain reactivity. Of Hall's original assumptions only two seem relevant to current usage. First, defecation is considered indicative of emotionality because it occurs in situations which are considered to provoke emotional responses. Second, defecation is assumed to be one of the responses stemming from autonomic innervation. Toback \& Schneirla (1962) have pointed out that this second assumption has received relatively little attention. In fact, studies of autonomic function associated with defecation in nonoperated, undrugged rats seem nonexistent. It was the purpose of this study to explore some of the details of defecation (DEF) behavior in different strains together with a different autonomic function, heart rate (HR).

\section{Method}

Ss were six male and five female Maudsley Nonreactive (MNR) and seven male and six female Maudsley Reactive (MR) rats in the 27 th inbred generation drawn from the colony of this laboratory. All animals were handled extensively from infancy and tested at 210 days of age or older.

Testing used Broadhurt's (1957) standardization of the open field test: number of fecal boli for $2 \mathrm{~min}$. a day on four successsive days in a $32-1 / 2$ in. uniform white circular stimulus field at $78 \mathrm{~dB}$ and $165 \mathrm{cp}$. HR was obtained with an electrode method similar to that of Ferrare, Silver, \& Snapper (1965) recorded on a Tektronix 564 oscilloscope in a raster display and photographed.

$\mathrm{DEF}$ in each of three 2-min. periods daily was recorded. The first of these was while the $S$ was in hand at its home cage location in the main animal room, the second 3 to $5 \mathrm{hr}$. later while being handled in the experimental room, and the last immediately thereafter in the open field. HR was recorded in the hand at the home cage location and in the open field. Testing order of animals was alternated from day to day.

Resulis

For statistical purposes nonparametric tests seemed most appropriate since conventional normalizing transformations are questionable where the interest is in the interrelationship of variables. Used here are the Wilcoxon-Mann-Whitney U for group differences and Page's $\mathrm{L}$ for monotonic trends or, with appropriate inversion, riionotonic contramovement.

The basic genetic difference of higher DEF in MRs was observed over all conditions $(U=10, p<.001)$. HR was lower for MRs over all conditions $(U=20, p<.01)$.

From handling at home cage to handling in the experimental room to open field, DEF declined slightly for MR and increased considerably for $\operatorname{MNR}(L=300, p<$ $.05)$. The same contramovement trend was found in $\mathrm{HR}$ differences from home cage handlin; to open field $(\mathrm{U}=12, \mathrm{p}<.001)$.

A monotonic contramovement trend over days was also observed in open field with MR DEF increasing and MNR DEF decreasing ( $L=704, p<.001)$. HR over days declined for both strains but much more markedly for MR (MR: $L=358, p<.001$; MNR: $L=141, p<.05$ ).$^{2}$

Dividing sessions into four $1 / 2-$ min. segments a decreasing HR for MRs was found for open field $(L=354$, $p<.01)$. There were no other within day trends.

Average rank difference correlation between DE F and HR was .11.

In summary, reactive animals showed higher DEF and lower HR than nonreactives. Compared with hand holding at the home cage, open field stress produced a slight decrement in DEF and marked HR deceleration in reactives and a marked increment in DEF and slight HR acceleration in nonreactives. Over days reactives showed increasing DEF and decreasing HR while nonreactives showed decreasing DEF and a slightly decreasing HR. Within days reactives show HR deceleration in the open field. Despite the HR-DEF group interactions, no correlation was observed over individual Ss.

\section{Discussion}

The data raise more questions than they answer. Both the defecation criterion on which selection was based and the $H R$ data fit the designations of reactive and nonreactive. There is, however, an implicit assumption that the defecation measures a stress reaction, i.e., reactive compared with nonreactive animals should show proportionately greater defecation differences between 
no stress and stress conditions. The data indicate that the opposite is the case. Also, in some cases defecation and HR trends show significant contramovement and in others parallelism. The behavior would seem to involve relatively specific and independent autonomic functions. The terms reactive and nonreactive should be interpreted strictly with reference to defining operations and not generalized to loose "emotionality" constructs.

Several theoretical alternatives can be considered. In order to produce homozygosity, selective inbreeding rather than individual selection was used in the development of these strains. While such a procedure has marked advantages for many genetic purposes it also has a higher prob): (h)ility of accidental fixation of unrelated genetic factors. Breeding experimentation would be required to resolve this possibility.

Since breeding selection was based on four days of testing it would tend to select high defecation and rising defecation against low defecation and declining defecation. If the increase and decline are defined as sensitization and adaptation, then the differentiation is that of sensitizers and adapters which could he independent of though here coupled with absolute defecation levels. If defecation is a cholinergic function there may also be a differentiation into parasympathetic and sympathetic reactors. This interpretation would fit the data generally but encounters some difficulty with the slight defecation decrement and major HR decrement from hand-home cage to open field. Levine (1960) suggested that handling may be a major stressor. From this viewpoint the extensive handling possibly could have led to $\mathrm{HR}$ deceleration habituation but not defecation adaptation in MRs.

For appropriate mechanisms Graham \& Clifton (1966) have clarified some of the apparent differences in the work of the Lacey's and Sokolov. In a Pavlovian framework it can be suggested that MRs show a dominant orienting reflex while MNRs have a dominant defense reflex. These are respectively stimulus enhancing and stimulus reducing. Under stress the MNR shows a defecatory stress response and the $\mathrm{HR}$ acceleration of the defense reflex. Stimulus effects are reduced facilitating habituation. For MRs handling is a stressor to which HR has habituated. Under a sufficiently severe stress, open field, the HR deceleration of the dominant orienting reflex enhances the stressor enhancing the dominant response, thus setting up a circular facilitation effect with increasing defecatory response and $\mathrm{HR}$ deceleration.

\section{References}

Bindra, D., \& Thompson, W. R. An evaluation of defecation and urination as measures of fearfulness. J. comp. physiol. Psychol., $1953,46,43-45$.

Broadhurst, P. L. Determinants of emotionality in the rat: I. Situtional factors. Brit. J. Psychol., 1957, 48, 1-12.

Broadhurst, P. L. Experiments in psychogenetics: Application of biometrical genetics to the inheritance of behavior. In $\mathrm{H}$. J. Eysenck (Ed.), Experiments in personality, Vol. I, Psychogenetics and psychopharmacology. London: Routledge \& Kegan Paul, 1960. Pp. 1-102.

Ferrare, D. P., Silver, M. P., \& Snapper, A. G. A method for cardiac recording from surface electrodes in the rat during free-operant procedures. J. exp. Anal. Behav., 1965, 8, 17-18.

Graham, F. K., \& Clifton, R. K. Heart-rate change as a component of the orienting response. Psychol. Bull., 1966, 65, 305-320.

Hall, C. S. Emotional behavior in the rat: I. Defecation and urination as measures of individual differences in emotionality. $J$. comp. Psychol., 1934, 18, 385-403.

Hall, C. S. The genetics of behavior. In S. S. Stevens (Ed.), Handbook of experimental psychology. New York: Wiley, 1951. Pp. 304-330.

Hunt, H. F., \& Otis, L. S. Conditioned and unconditioned emotional defecation in the rat. J. comp. physiol. Psychol., 1953, 46, 378383.

Lebo, D. A simplified method for measuring emotional defecation in the rat. Science, 1953,118, 352-353.

Levine, S. Stimulation in infancy. Scient. American, 1960, 201 (5), 81-86.

Lindzey, G., Winstone, H. D., \& Whitney, G. D. Defecation in stressful situations. Psychon. Sci., 1964, 1, 3-4.

O'Kelly, L. I. The validity of defecation as a measure of emotionality in the rat. J. gen. Psychol., 1940, 23, 75-87.

Pare, W. P. Relationship of various behaviors in the open-field test of emotionality. Psychol. Rep., 1964, 14, 19-22.

Tobach, E., \& Schneirla, T. C. Eliminative responses in mice and rats and the problem of emotionality. In E. L. Bliss (Ed.), Roots of behavior. New York: Hoeber, 1962. Pp. 211-231.

Yoshioka, J. G. Learning vs. skill in rats. J. genet. Psychol., $1932,41,401-416$.

\section{Notes}

1. The second author is now a graduate student at the University of New Mexico. This study was carried out in partial fulfillment of requirements for the $B$. A. under supervision of the first author. 2. Because of a partial data loss suffered on a film of first day MNR HR records, the MNR daily $\mathrm{HR}$ trend test is based on only the last three days. 